\title{
Essais
}

ESSAIS

Revue interdisciplinaire d'Humanités

\section{« Hirvi Dharti, Stri Shakti, Manav Murti »}

(«Terre verte, puissance féminine, libération humaine ») Ce que le féminisme apporte à l'écologie

Jeanne Burgart Goutal

\section{OpenEdition}

1 Journals

Édition électronique

URL : http://journals.openedition.org/essais/461

DOI : 10.4000/essais.461

ISSN : 2276-0970

Éditeur

École doctorale Montaigne Humanités

Édition imprimée

Date de publication : 1 janvier 2018

Pagination : 81-96

ISBN : 979-10-97024-03-1

ISSN : 2417-4211

Référence électronique

Jeanne Burgart Goutal, « «Hirvi Dharti, Stri Shakti, Manav Murti » », Essais [En ligne], 13 | 2018, mis en ligne le 01 décembre 2019, consulté le 12 décembre 2019. URL : http://journals.openedition.org/ essais/461 ; DOI : 10.4000/essais.461 


\section{La métaphore du viol de la terre : une proposition écoféministe}

Noémie Moutel

Les linguistes-cognitivistes Mark Johnson et George Lakoff affirment que " notre système conceptuel ordinaire, qui nous sert à penser et à agir, est de nature fondamentalement métaphorique ${ }^{1}$ ". Selon eux, la métaphore est une forme d'expression qui n'émane pas seulement de notre expérience du réel : elle influence aussi la représentation que nous en avons. Ainsi, la métaphore n'est pas limitée à " un procédé de l'imagination poétique et de l'ornement théorique ${ }^{2}$ ", mais désigne plutôt un procédé de représentation du réel culturellement construit et transmis. Par exemple, Johnson et Lakoff qualifient l'expression « le temps, c'est de l'argent » de concept métaphorique, car son usage n'effectue pas une simple comparaison entre le temps et l'argent mais traduit un cadre culturel spécifique. En l'occurrence, la culture occidentale moderne est régie par l'idéologie du capitalisme financier, qui structure un cadre social où les tâches sont salariées à l'heure ou à la journée, et où le temps de travail est comptabilisé et proportionnellement indemnisé.

Ces pratiques sont apparues dans les sociétés modernes industrialisées et structurent très profondément nos activités quotidiennes fondamentales. Du fait que nous agissons comme si le temps était une denrée précieuse et une ressource limitée, comme si même c'était de l'argent, nous concevons le temps de cette manière. Ainsi, nous comprenons et vivons le temps comme quelque chose qui peut être dépensé, perdu, calculé, bien ou mal investi, épargné ou gaspillé3.

Une métaphore « permet de comprendre quelque chose (et d'en faire l'expérience) en termes de quelque chose d'autre ${ }^{4}$ ». Lorsqu'une métaphore est culturellement pertinente et globalement comprise et utilisée, elle constitue un concept métaphorique, qui reflète un réseau systématique d'expressions et de comportements associés.

1 Mark Johnson et George Lakoff, Les métaphores dans la vie quotidienne, trad. Michel de Fornel et Jean-Jacques Lecercle, Paris, Éditions de Minuit, 1985, p. 13.

2 Id.

3 Ibid., p. 18.

4 Ibid., p. 15. 
Le concept métaphorique que cet article propose d'étudier est celui du viol de la terre. Bien qu'on retrouve sa trace dans des textes de l'Antiquité grecque et romaine, la métaphore du viol de la terre a cessé de qualifier les pratiques d'extraction minière motivées par le mercantilisme des hommes blancs depuis l'aube de la Renaissance. Ce concept métaphorique est donc relativement nouveau, car son actualisation est en cours, et cet article entend y participer : «notre conviction est que les métaphores nouvelles donnent du sens à nos expériences, de la même manière que les métaphores conventionnelles ${ }^{5}$ ». Afin de structurer et de clarifier le concept métaphorique que nous souhaitons étayer, nous l'énoncerons comme suit : l'agriculture et l'extraction minière industrielles perpétuent le viol de la terre.

$\mathrm{Au}$ XVII ${ }^{e}$ siècle, en Angleterre, un homonyme désignait à la fois la notion de terre, au sens de territoire, et la notion de viol, au sens de pénétration non consentie du corps de l'autre. En effet, le nom commun rape désignait les districts administratifs du Sussex ${ }^{6}$, délimités grâce à une corde tendue, rope en anglais. Le même terme décrivait déjà l'acte que nous qualifions de viol en français. On remarque alors que le territoire, dont les limites peuvent être transgressées, et le corps féminin, sujet lui aussi à l'intrusion, sont sémantiquement liés par un terme désignant à la fois la zone délimitée et l'agression sexuelle. Il existe également en français une proximité linguistique entre le viol du corps et celui de la propriété, terrienne ou immobilière, que l'on observe dans le terme violation, du latin violare, transgresser, profaner, ou désacraliser, lui-même issu de la racine latine vis, signifiant force. Violer signifie donc : faire usage de la force pour posséder une terre ou une personne, transgresser et profaner les limites qui la définissent et, ce faisant, la désacraliser.

C'est à la lumière des constats écoféministes contemporains, qui soulignent la consubstantialité de l'exploitation des femmes et des ressources naturelles, qu'il nous semble pertinent d'observer la récurrence de la métaphore du viol de la terre dans la pensée occidentale. Il s'agit ainsi de rendre saillant un mode de pensée et d'action caractéristique du néo-libéralisme blanc et patriarcal. Le développement de l'agriculture intensive et de l'extraction industrielle de ressources minières et fossilisées s'appuie sur des représentations culturelles qui normalisent le cadre éthique d'une transgression constante des limites physiques et ontologiques du vivant. En observant les mutations de la métaphore culturelle du viol de la terre, en lien avec la thématique de l'appropriation du vivant dans les sociétés occidentales, cet article entend participer à l'enrichissement de nos modes de réflexion et d'action vis-à-vis des féminismes et de l'écologie.

5 Ibid., p. 149.

6 C.T. Onion, R.W. Burchfield et G.W.S. Friedrichsen, The Oxford Dictionary of English Etymology, 1966. 


\section{Personnification de la terre}

Pour Johnson et Lakoff, la métaphore est essentielle au langage et à la conceptualisation de la réalité car elle donne une substance à ce qu'il est difficile de définir ou de délimiter. La représentation culturelle de la planète en tant qu'être vivant est une personnification récurrente dans les mythologies et philosophies des sociétés antiques occidentales. Dans un article intitulé " Mining the Earth's Womb ", où elle synthétise un des arguments principaux de son ouvrage The Death of Nature, Woman, Ecology and the Scientific Revolution, Carolyn Merchant avance que cette personnification, comme tout concept métaphorique répandu, est doté d'un caractère normatif qui conditionne les agissements humains :

L'image de la terre comme organisme vivant et mère nourricière a tenu lieu de contrainte culturelle limitant l'action des êtres vivants. On ne poignarde pas aisément sa mère, ni ne fouille ses entrailles pour y chercher de l'or, ni ne mutile son corps [...] Aussi longtemps que la terre était considérée comme vivante et sensible, on pouvait regarder les actes de destruction à son encontre comme une violation de l'éthique comportementale humaine. Pour la plupart des cultures traditionnelles, les minéraux et les métaux mûrissaient dans l'utérus de la Terre-Mère, les mines étaient comparées à son vagin et la métallurgie était la précipitation humaine de la naissance des métaux vivants dans le ventre artificiel de la fournaise - un avortement avant terme du cycle de croissance naturelle des métaux. Les mineurs cherchaient la rédemption auprès des divinités du sol et du monde souterrain en exécutant des cérémonies sacrificielles, en observant une propreté stricte, une abstinence sexuelle, et ils pratiquaient le jeûne avant de violer le caractère sacré de la terre vivante en descendant dans la mine.

Philosophe des sciences, Carolyn Merchant remarque que l'imagerie culturelle de la terre comme mère structurait un cadre déontologique spécifique : "des auteurs romains comme Sénèque, Ovide et Pline, ainsi que les Stoïciens, déploraient ouvertement l'exploitation comme un viol de leur mère, la terre ${ }^{8}$ ».

L'approche féministe de l'histoire et de la philosophie des sciences occidentales de Carolyn Merchant démontre que la pratique de l'extraction de ressources géologiques est objet de réflexion et de controverse depuis plus de deux millénaires. Si nous souhaitons réveiller la métaphore du viol de la terre à l'aube du XXI' siècle, c'est afin de participer aux efforts de conceptualisation des conditions culturelles qui non seulement encadrent, mais participent des changements climatiques de l'ère de l'Anthropocène. En ce sens, l'hypothèse

7 Carolyn Merchant, "Mining the Earth’s Womb ", in Émilie Hache (éd.), Reclaim. Recueil de textes écoféministes, trad. Émilie Notéris, Paris, Éditions Cambourakis, "Sorcières ", 2016, p. $131-132$. 
Gaïa formulée par les géologues James Lovelock, John Epton et Lynn Margulis ${ }^{9}$ est une actualisation contemporaine de l'ancienne métaphore culturelle de la personnification de la planète Terre en tant que mère nourricière.

Dans une perspective historique, l'importance des métaphores culturelles apparaît à l'endroit même où celles-ci se transforment et décrivent autrement certains phénomènes, induisant ainsi de nouvelles contraintes comportementales. Carolyn Merchant soutient l'idée que la métaphore culturelle de la terre en tant qu'être féminin vivant, nourricier et sensible perdure, dans l'imagerie occidentale, de l'Antiquité jusqu'à l'orée de la Renaissance. Elle interroge les textes philosophiques et littéraires du $\mathrm{XV}^{\mathrm{e}}$ siècle à la recherche d'éléments indiquant des transformations dans les métaphores répandues pour représenter la terre, afin d'analyser comment le déploiement d'une philosophie mercantile influence ces représentations. L'imagerie culturelle fonctionne alors de manière cyclique, car les nouvelles métaphores influencent à leur tour l'éthique et les valeurs de la société qui les utilise, donnant lieu à des activités, des expériences et des échanges qui appelleront d'autres représentations.

\section{Métaphore culturelle et appropriation du vivant : la terre mère devient corps de femmes}

Quelques vers d'un poème de John Donne, «Le coucher de sa maîtresse », donnent à lire comment la métaphore culturelle de la terre nourricière mute d'une personnification d'un être maternel et sacré à un corps sexuellement désirable. La métaphore opère alors telle une approbation des nouvelles pratiques commerciales, en l'occurrence la colonisation du continent nord-américain et l'extraction de ses ressources minières :

\footnotetext{
Laisse, laisse quêter ma main buissonnière

Par-dessus, par-dessous, entre, devant, derrière !

Terre-Neuve ! Amérique ! Ô ma possession,

Qu'un seul homme garnit mieux qu'une garnison!

Ma mine de pierres précieuses! Mon Empire,

Dont l'exploration m'est bienheureux délire ${ }^{10}$ !
}

Ces vers montrent que la mutation de la représentation de la terre est directement liée à la colonisation de nouveaux territoires par le patriarcat blanc. L'amante à qui s’adresse la voix poétique se nomme "Terre-Neuve ", "Amérique ". Elle doit se laisser explorer, se laisser posséder et se laisser garnir... La métaphore de l'exploration du corps féminin qu'utilise John Donne pour décrire la conquête de nouveaux territoires crée une similitude entre la corporéité féminine et un espace géographique en cours d'appropriation. Sous sa plume, les prépositions de

9 John Epton, James Lovelock et Lynn Margulis, "The Quest for Gaïa ", in The New Scientist, vol. 65, n 935,6 février 1975.

10 John Donne, Poèmes, trad. Yves Denis et Jean Fusier, Paris, Gallimard, 1962, p. 112. 
lieux, qui composent entièrement le deuxième alexandrin, se prêtent autant au champ lexical de la sexualité qu'à celui de l'extraction minière et de la conquête territoriale.

Johnson et Lakoff insistent sur le fait que les similitudes exprimées par l'usage de la métaphore ne sont pas nécessairement pré-existantes à la comparaison qui s'opère. La similitude du corps et du territoire exploré n'est pas inhérente aux éléments liés par la métaphore, mais au contraire créée par le propos subjectif articulé par Donne. C'est l'expérience subjective du réel, et le contexte culturel dans lequel elle s'inscrit, qui construit l'expression métaphorique et « détermine les catégories de notre système conceptuel [...]. Le seul type de similitude pertinent pour les métaphores est d'ordre expérientiel et non objectif [...]. Les métaphores conceptuelles sont fondées sur des corrélations dans notre expérience ${ }^{11}$. »

Dans The Lay of the Land, Metaphor as Experience and History in American Life and Letters, publié cinq ans avant l'ouvrage de Johnson et Lakoff, Annette Kolodny observait déjà comment la métaphore de la-terre-comme-femme - «land-aswoman» - caractérise les motivations et les comportements des colonisateurs blancs lors de leur expansion territoriale en Amérique du Nord : " telle que je l'utilise, la métaphore de la terre-comme-femme implique un groupe varié de structures de mots (habituellement exprimé comme des images), qui à maintes reprises affirment la réalité expérientielle d'un objet particulier - dans ce cas, la réalité intrinsèquement féminine du vaste paysage américain ${ }^{12}$ ".

L'apport majeur de la démonstration de Kolodny tient en ce qu'elle rend ostensible le cadre culturel patriarcal qui conditionne la mise en ouvre du processus de colonisation. Pour Kolodny, l'impulsion pastorale américaine est liée à la métaphore du territoire en tant que corps féminin. Elle observe, dans les écrits des explorateurs des XVI ${ }^{e}$ et XVII ${ }^{e}$ siècles, tels qu'Arthur Barlowe et John Rolfe, que la corrélation entre topographie du territoire et anatomie féminine anime les récits de la découverte du Nouveau Monde.

La pastorale européenne s'exporte vers des terres mystérieuses, propices à la projection de fantasmes, de rêves et de désirs. Les similitudes qui sont créées, entre corps féminin et nouveaux territoires, produisent alors un discours sur la démarche coloniale en elle-même : " qu'il s'agisse de politique nationale ou d'interaction quotidienne, ceux qui possèdent le pouvoir réussissent à imposer leurs métaphores ${ }^{13}{ }^{\prime}$. Exposer la constance de la métaphore de la terre-commefemme revient à indiquer que ceux qui possèdent le pouvoir colonisateur entretiennent, par le langage, le système patriarcal dans lequel ils évoluent.

11 Mark Johnson et George Lakoff, Les métaphores dans la vie quotidienne, op. cit., p. 164.

12 Annette Kolodny, The Lay of the Land. Metaphor as Experience and History in American Life and Letters, University of North Carolina Press, 1975, p. 150, trad. personnelle (sauf mention contraire, tous les textes traduits qui suivent le sont par l'auteure).

13 Mark Johnson et George Lakoff, Les métaphores dans la vie quotidienne, op. cit., p. 168. 
Dans la plupart des cas, ce n'est pas la vérité ou la fausseté d'une métaphore qui est en jeu, mais les perceptions et inférences qui s'autorisent d'elle, ainsi que les actions qu'elle sanctionne. Dans notre vie la plus quotidienne, [...] nous définissons la réalité en termes de métaphores et nous agissons en fonction de celles-ci. Nous faisons des inférences, nous fixons des objectifs, nous prenons des engagements et nous exécutons des plans. Nous le faisons sur la base d'une structuration consciente ou inconsciente de notre expérience à partir de métaphores ${ }^{14}$.

Annette Kolodny qualifie sa recherche d'enquête psycho-historique, dans le sens où l'historicité de l'usage de la métaphore de la terre-comme-femme indique quelles dispositions psychologiques et quels modes de représentation de la réalité ont organisé et sous-tendu la mise en place de ce qui deviendrait les États-Unis d'Amérique. Sa démonstration de la puissance et de l'omniprésence de la métaphore de la terre-comme-femme dans les lettres nord-américaines a pour but de rendre saillants les mécanismes psychologiques à l'œuvre dans l'expansion, l'occupation et l'exploitation territoriale dont la mouvance écologiste des années 1970 dénonce le caractère destructeur, abusif et absurde. Si Kolodny ne s'inscrit pas explicitement dans la pensée écoféministe, la mise en lumière de la consubstantialité des présomptions hégémoniques blanches et patriarcales dans la colonisation du territoire nord-américain nourrit indubitablement l'analyse écoféministe.

L'étude minutieuse que constitue The Lay of the Land: Metaphor as Experience and History in American Life and Letters précède le terme de " critique littéraire écoféministe " mais elle n’en contient pas moins tous les éléments. En retraçant la période historique du début de la colonisation américaine jusqu'à l'année de l'élection d'Abraham Lincoln (fin XVI jusqu'à fin XIX ${ }^{\mathrm{e}}$ siècles), l'œuvre de Kolodny est la première à tenter de cerner les retombées de cette association conceptuelle, anciennes et actuelles, sur la crise écologique contemporaine en se concentrant sur les références faites à la terre américaine comme étant une " entité » féminine ${ }^{15}$.

Nous souhaitons ici poursuivre le travail sur la métaphore de la terrecomme-femme initié par Merchant et Kolodny en y ajoutant un élément qui caractériserait le comportement d'une société patriarcale blanche vis-àvis d'une terre métaphoriquement conçue comme un corps féminin passif, offert à l'exploration et à l'ensemencement. La notion de viol désigne l'intrusion d'un corps dans un autre, et la métaphore du viol de la terre permet d'interroger le rapport ontologique que les sociétés modernes entretiennent avec le vivant, c'est-à-dire avec un ensemble de formes de vie qualifiées de non-humaines.

14 Id.

15 Margot Lauwers, Amazones de la plume, les manifestations littéraires de l'écoféminisme conteporain, thèse de doctorat en études anglophones, Université de Perpignan, 2014, p. 81. 


\section{La Révolution scientifique : contraindre et asservir le vivant}

Aux XVI ${ }^{e}$ et XVII ${ }^{e}$ siècles, la Révolution scientifique permet des avancées technologiques qui évoluent parallèlement au commerce triangulaire et au développement de l'extraction minière à grande échelle. S'affirme alors une relation au vivant radicalement éloignée de la révérence, de la précaution et des considérations éthiques que générait la personnification antique de la terre en tant que mère nourricière. La philosophie mercantile qui structure la phase d'accumulation primitive du capitalisme accorde aux ressources minières et à leur extraction de nouvelles fonctions et significations : "si les nouvelles valeurs connectées à l'exploitation minière étaient positives [...] alors la comparaison pouvait être retournée. Autoriser l'exploitation minière autorisait le viol ou l'exploitation technologique de la terre. Le contexte organique était littéralement sapé par la nouvelle activité commerciale ${ }^{16}$ ».

Cette modification dans le rapport éthique entre humains et formes de vie non-humaines s'effectue au long de plusieurs décennies, et nous ne saurions déterminer spécifiquement si l'activité commerciale, la philosophie mercantile ou la méthodologie scientifique initie cette mutation. Nous pouvons toutefois constater l'influence cruciale des travaux de Francis Bacon dans la mise en place de représentations culturelles du vivant qui valident l'idée d'une domination indiscutable des humains sur toutes les formes de vie non-humaines. En effet, il encourage une éthique scientifique intrusive et dominatrice, en affirmant que la nature doit être " réduite en servitude ", et traitée comme " une esclave ", mise " sous contrainte ", et " modelée " par les arts mécaniques. Bacon préconise de disséquer la nature, de découper la matière et de placer la techné au-dessus de la nature naturante.

Dans $O f$ the Dignity and Advancement of Learning, Bacon compare la nature à " une vulgaire catin ${ }^{17}$ ", et affirme que dominer la nature est une mission d'ordre divin dont les hommes sont investis. L'assujettissement de la nature aux velléités hégémoniques d'hommes revendiquant une légitimité religieuse est corrélée, historiquement et culturellement, à la période de l'Inquisition. La rédaction et la diffusion du Malleus malleficarum, qui diabolise la liberté sexuelle des femmes, leur autonomie et leur intimité, use d'une même rhétorique que celle par laquelle Bacon présente la nature comme un être dont il faut dévoiler les secrets et déjouer les complots. Pour les inquisiteurs Heinrich Kramer et Jakob Sprenger ${ }^{18}$, comme pour Francis Bacon, les

16 Carolyn Merchant, « Mining the Earth's Womb », op. cit., p. 153.

17 Tous les termes attribués à Francis Bacon sont cités par Carolyn Merchant dans « Mining the Earth's Womb ». op. cit., p. 153-155.

18 Heinrich Kramer et Johannes Sprenger, Malleus Maleficarum [1486], New York, Cosimo Inc, 2007. 
femmes et la nature requièrent que les hommes fassent preuve de force (vis), et imposent l'intrusion par un processus inquisiteur qui ne cherche à aucun moment le consentement de l'autre.

C'est ici, dans l'imagerie sexuelle brutale que réside la clé de la méthode expérimentale moderne - la contrainte de la nature en laboratoire, la dissection manuelle et mentale, et la pénétration des secrets cachés - langage encore employé de nos jours pour louer les "faits bruts », "l'intelligence pénétrante ", ou encore "l'idée-force " du scientifique. Les contraintes de l'exploitation minière de la terre se sont retournées en approbation langagière légitimant l'exploitation et le « viol» de la nature pour le bien des hommes ${ }^{19}$.

La période dite des Lumières représente, dans une lecture écoféministe des rapports entre les humains et la nature, une période de bouleversement des comportements vis-à-vis du vivant. Si la pensée pré-moderne et mécaniste se définit comme séparée de la nature, cette pensée est exprimée et actée par des institutions dont les femmes sont tout à fait exclues. Une corrélation peut donc être établie entre l'implacabilité et l'ampleur de la misogynie de l'époque et l'avènement du rationalisme. Johnson et Lakoff n'appliquent pas de grille de lecture féministe à leur analyse de l'usage de la métaphore, mais précisent comment la première révolution industrielle a initié une distinction clivante entre des expériences dites subjectives et des vérités dites objectives. Pour eux, le mythe de l'objectivité scientifique contribue à nier l'importance des réseaux métaphoriques qui conceptualisent notre appréhension de la réalité.

Lorsque la science, par le biais de la technologie, devint toute-puissante, et que la Révolution industrielle imposa une réalité inhumaine, une réaction se produisit parmi les poètes, les artistes et les philosophes : c'est l'origine de la tradition romantique. [...] La science, la raison et la technologie avaient aliéné l'homme, par rapport à lui-même et à son environnement naturel. Les romantiques voyaient dans la poésie, l'art et le retour à la nature les moyens de reconquérir son humanité perdue. [...] La tradition romantique, en adoptant le point de vue subjectiviste, renforce la séparation entre la vérité et la raison, d'un côté, et l'art et l'imagination, de l'autre ${ }^{20}$.

Le roman de Mary Shelley, Frankenstein ou le Prométhée moderne, ouvrage de référence de la tradition romantique, est publié à l'époque de la jonction historique dont font état Johnson et Lakoff. Le roman rassemble, par son récit et les conditions de son écriture, les caractéristiques écoféministes qui nous occupent. Écrit par une femme à l'époque où signer une œuvre littéraire pouvait nuire durablement à sa réputation, le texte met en évidence les dangers d'une méthodologie scientifique qui tend à s'émanciper de la cyclicité organique de la reproduction du vivant.

19 Carolyn Merchant, "Mining the Earth's Womb ", op. cit., p. 157.

20 Mark Johnson et George Lakoff, Les métaphores dans la vie quotidienne, op. cit., p. 203. 
Theodore Roszak, dans Person/Planet, postule que le roman de Mary Shelley est inspiré par des considérations proto-écologistes. Pour lui, le passage qui illustre le plus clairement le drame d'une recherche scientifique dépourvue de déontologie écologique est celui où Victor, quelques heures après avoir donné vie à sa créature, rêve d'Elizabeth.

Je dormis, il est vrai, mais d'un sommeil troublé par les rêves les plus terribles. Je croyais voir Elizabeth, dans la fleur de sa santé, passer dans les rues d'Ingolstadt. Délicieusement surpris, je l'embrassais ; mais à mon premier baiser sur ses lèvres, elles revêtaient la lividité de la mort ; ses traits paraissaient changer, et il me semblait tenir en mes bras le corps de ma mère morte ; un linceul l'enveloppait, et je vis les vers du tombeau ramper dans les plis du linceul. Je tressaillis et m'éveillai dans l'horreur ; une sueur froide me couvrait le front, mes dents claquaient, tous mes membres étaient convulsés : c'est alors qu'à la lumière incertaine et jaunâtre de la lune traversant les persiennes de ma fenêtre, j’aperçus le malheureux, le misérable monstre que j'avais créé2 ${ }^{1}$.

Pour Theodore Roszak, ce passage est représentatif des excès que la recherche scientifique engendre, et de la froideur et de l'insensibilité de la méthodologie scientifique patriarcale. Il voit dans le personnage rêvé par Victor, qui revêt tour à tour l'apparence de sa cousine et fiancée, puis de sa mère, une représentation de la nature, dont la mort est intériorisée par Victor. La femme que le Prométhée moderne tient dans ses bras est à la fois mère de chair et Mère Nature, "tuée par sa prétention impitoyable et masculine à la domination. [...] Ainsi elle meurt pour lui - elle meurt à l'intérieur de lui ${ }^{22}$ ".

Le rêve de Victor donne à voir ce qu'intuitivement, dans sa fièvre postcréation, il sait avoir détruit. Et l'interprétation de l'œuvre laisse bien souvent de côté les implications de ce rêve, car l'idéologie du progrès et le mythe de l'objectivité nous imprègnent tant que nous échouons à accorder de la crédibilité aux visions oniriques décrites par Shelley.

Obsédés par les nombres, les objets, la propriété privée et la force physique nous avons appris à nier les faits de nos sentiments, la réalité de nos forces intuitives. Nous séparons le "dedans " du " dehors " et puis nous dénigrons le subjectif, insistant que ce n'est qu'un fantasme, arbitraire et inventé. C'est ainsi que nous nous rendons sourds à la voix du sacré, au langage de la Terre. [...] Et si l'on pouvait, au contraire, entendre la voix de la planète dans le sens personnel que nous attribuons au monde? Après tout, où le Dr. Frankenstein découvre-t-il la vérité à propos de lui-même et de son travail ? Dans un rêve... et puis... trop $\operatorname{tard}^{23}$.

21 Mary Shelley, Frankenstein ou le Prométhée moderne [1831], trad. Germain d'Hangest, Paris, Flammarion, 1997, p. 78.

22 Theodore Roszak, Person/Planet, Londres, Paladin books, 1978, p. 71.

23 Ibid., p. 71-72. 
L'expression de réalités subjectives est le moyen par lequel le dialogue entre personnes peut se renouveler et se déployer, donner naissance à de nouvelles compréhensions et acceptations de nos liens avec l'environnement. L'interprétation que Roszak propose du cauchemar qui hante le sommeil de Victor Frankenstein dans les heures qui suivent l'éveil de la créature qu'il a amenée à la vie corrobore les perspectives de Johnson et Lakoff au sujet du statut du langage métaphorique depuis l'essor du principe de rationalité scientifique. Le concept métaphorique du viol de la terre nous permet d'évoquer comment le comportement des sociétés occidentales industrielles patriarcales contemporaines ne violentent pas seulement des personnes ou des espaces géographiques, mais viole constamment le principe du continuum écologique, de la cyclicité et de la regénération du vivant.

\section{Carnophallogocentrisme et éco-révolution}

Le concept métaphorique du viol de la terre semble pertinent pour décrire le comportement des sociétés occidentales modernes vis-à-vis de l'environnement naturel, car le terme "viol " renvoie à la fois au féminin (dans le sens où l'immense majorité des victimes de viol sont des femmes et des filles) et à la propriété privée. Les sociétés patriarcales sont structurées grâce à un fonctionnement patrilinéaire, par le biais duquel le patronyme et le patrimoine d'une famille se transmettent de père en fils. À plus grande échelle, ce système d'imbrication de groupes patrilinéaires peut former une patrie. Le principe de primogéniture est culturellement actif depuis l'Antiquité, et contribue, à chaque génération, à l'effacement et à l'assujettissement des existences féminines.

L'œuvre de Susan Griffin, Woman and Nature, propose, par la juxtaposition de voix historiques, affirmées, considérées comme objectives, et de voix empathiques, sensibles et imaginatives, dites subjectives, de déconstruire le langage patriarcal par l'insertion littéraire et poétique d'autres points de vue. Son texte " cherche à mettre à distance le discours patriarcal contenu dans l'histoire de la philosophie occidentale ${ }^{24}$ ". Dans la première partie de Woman and Nature, dans la section "Land ", soit terre, ou sol, se trouve un passage intitulé Primogeniture. Nous le reproduisons ici, avec la même typographie, c'est-à-dire une voix en lettres romaines et droites, et une autre en italiques, forme choisie par Susan Griffin pour construire une prose hors du commun.

24 Note de l'éditrice Isabelle Cambourakis à propos de Susan Griffin, «Où sont exposées conjointement et chronologiquement les idées de l'homme à propos de la nature et des femmes ", dans Reclaim, op. cit., p. 59. 
Primogéniture.

En droit, loi sur l'héritage selon laquelle la terre revient au fils aîné.

Nous allons vous dire qui a gagné et qui a perdu de cette façon car il y en avait, les pères, qui détenaient savaient que leurs noms et d'autres qui ne détenaient pas perdureraient ces êtres qui étaient connus, que les grands domaines et ces êtres attestant de leur gloire et de leur renommée qui étaient inconnus perdureraient ces êtres dont les vies furent effacées et le pouvoir qui émanait de ces propriétés terriennes ces êtres dont le labeur continuerait, génération après génération comme le labeur des champs, de la terre, de grandir dans les esprits des vivants passerait comme le souffle qui quitte les vivants ${ }^{25}$.

Si l'on rassemble les passages en italique, on obtient un propos, distinct et pourtant imbriqué dans celui de la typographie usuelle: "Nous allons vous dire qui a gagné et qui a perdu, car il y en avait qui détenaient, et d'autres qui ne détenaient pas, ces êtres qui étaient connus, et ces êtres qui étaient inconnus, ces êtres dont les vies furent effacées, ces êtres dont le labeur, comme le labeur des champs, de la terre, passerait comme le souffle qui quitte les vivants. " La voix en italique est celle de personnes oubliées, qui n'ont ni nom, ni terres, et dont l'œuvre, le travail, l'existence, disparaissent avec leur dernier souffle. En rapprochant les autres segments de l'énoncé, on accède à la voix qui demeure, celle, figée, écrite, pérenne, de l'histoire masculine : «De cette façon les pères savaient que leurs noms perdureraient et que les grands domaines attestant de leur gloire et de leur renommée perdureraient et que le pouvoir qui émanait de ces propriétés terriennes continuerait, génération après génération de grandir dans les esprits des vivants ". L'imbrication de ces deux voix nous amène dans un espace dual, ambivalent et ambigu, où l'on est poussé à entendre des voix discordantes.

La prose de Susan Griffin exige alors que nous pensions différemment. Il s'agit de transformer les rapports concrets entre les êtres, et l'autorité historique et linguistique à leur disposition, en transformant tout d'abord les rapports de conscience. Le féminisme radical et l'écologie profonde soutiennent qu'un certain type de conceptualisation imprègne nos structures occidentales. Celle-ci est identifiée comme une structure dualiste, patriarcale et anti-naturaliste, qui dévalorise les femmes et les animaux. Pour Jacques Derrida, cette conscience peut être caractérisée de carnophallogocentrique, un nœud inextricable construit de trois travers. Le premier, signifié par le préfixe carno-, est spéciste, c'est-à-dire anthropocentriste, concevant que l'espèce humaine est supérieure à toutes les autres espèces. Le deuxième, signifié par phallo-, dénote le sexisme d'une société patriarcale. Le troisième, signifié par logo-se rapporte au principe rationalisant du langage. Cette architecture semble imperturbable : les humains dominent les autres espèces, les hommes dominent les femmes, et le langage domine la raison ${ }^{26}$.

25 Susan Griffin, Woman and Nature, The Roaring Inside Her, New York, Harper \& Row, p. 52.

26 Jacques Derrida, L'animal que donc je suis, Paris, Galilée, 2006, p. 72-83. 
Jeanne Burgart-Goutal, dans sa communication « Penser différemment : le défi du langage écoféministe " (colloque international d'écopoétique de Perpignan en 2015), constate la prévalence, dans la philosophie occidentale, d'un déni d'existence ontologique des femmes et des animaux, et de leur capacité à s'exprimer. Réputés muets, les femmes et les êtres naturels sont exclus du groupe des sujets parlants. Les mots sont, selon Jacques Derrida et Jeanne Burgart-Goutal, inadéquats, car porteurs de biais carnophallogocentriques. La domination est donc consubstantielle de la nomination.

\section{Statut légal du viol et justice environnementale}

Le concept métaphorique du viol de la terre met en lien la misogynie des sociétés occidentales et l'exploitation irraisonnée des formes de vie nonhumaines. L'évolution du statut légal du viol, portée par les luttes féministes des années 1960 et 1970, s’impose au moment même où les luttes écologiques appellent à la prise en compte de la finitude des ressources planétaires, de la souffrance animale et de la déplétion des richesses organiques des sols. Nous voyons dans cette concomitance une transformation des métaphores culturelles liées aux femmes et formes de vie dites non-humaines.

Gina Filizzola et Gérard Lopez indiquent, dans leur ouvrage Le viol, que depuis la première table des lois - le code de Hammurabi, datant de la civilisation babylonienne - jusqu'au code civil de Napoléon, datant de 1803, le viol était sanctionné par la justice dans la mesure où il présentait un risque quant à la transmission patrilinéaire du patrimoine. Le statut du viol dans la loi pénale visait donc à " protéger la légitimité de potentielles grossesses ${ }^{27}$ ". Ce n'est qu'en 1980 que la législation française change : " il s'agit à présent de protéger l'intégrité morale de la victime : tout acte de pénétration est un $v^{v i o}{ }^{28}$ ». L'article 222-23 du Code Pénal stipule ainsi que " tout acte de pénétration sexuelle, de quelque nature qu'il soit, commis sur la personne d'autrui par violence, contrainte, menace ou surprise est un viol. Le viol est puni de 15 ans de réclusion criminelle ${ }^{29}$ ».

Les luttes actuelles visant à promouvoir la mise en ouvre d'une justice environnementale nous interpellent sur la façon dont nous pourrions étendre le droit aux autres formes de vie terrestres. La thèse défendue par Theodore Roszak dans Person/Planet est que " les besoins de la planète sont les besoins de la personne, et donc, les droits de la personne sont les droits de la planète ». Si l'on appliquait le code pénal actuel à cette thèse écologiste, quels actes pourraient relever d'un viol de la terre, d'un acte de pénétration

27 Gina Filizzola et Gérard Lopez, Le viol, Paris, PUF, 1993, p. 31.

28 Id.

29 Code Pénal, Paris, Dalloz, 2017, p. 620. 
non consenti commis sur une forme de vie minérale, végétale ou animale, par violence, contrainte, menace ou surprise ? Dans The Gendered Atom, Roszak approfondit son exploration des corrélations possibles entre le fonctionnement psychologique d'une personne coupable de viol, et le comportement d'une partie de l'humanité vis-à-vis du monde naturel.

Pour le violeur, la sexualité devient un moyen de compenser des sentiments sous-jacents d'inadéquation et sert à exprimer des problèmes de maîtrise, d'identité et de capacité. Son but est de capturer et de contrôler sa victime. C'est là que le "viol de la nature " cesse d'être une métaphore. C'est une description adéquate d'un certain fonctionnement psychologique qui produit de façon prévisible un certain type de comportement. Le principe de légitimité est à la base de la sexualité masculine standard. Jusque très récemment, c'està-dire jusqu'à ce que les femmes affirment leur droit à contrôler leur propre corps - l'homme s'octroyait le droit de sexualiser le corps de la femme, de le juger, de le commenter, de le choisir, de l'exciter, de s'en servir pour son plaisir, et de lui donner tout le plaisir que ce corps était censé souhaiter. Et là où l'on résistait à ce droit, l'homme se sentait autorisé à le prendre de force. Le même sentiment de légitimité gouverne notre relation au monde naturel. Pendant les quatre siècles passés à puiser richesses et confort du corps de la terre, la science moderne n'a pas pris la peine d'inventer un seul rite ou rituel, pas même la moindre prière qui demanderait pardon ou rendrait grâce. Mais après tout, quel sens y aurait-il à demander quoi que ce soit d'un corps mort ${ }^{30}$ ?

Dans Solutions Locales pour Désordre Global, Claude et Lydia Bourguignon, ingénieurs agronomes et fondateurs du Laboratoire d'Analayses Microbiologiques des Sols, expliquent que le labour profond est, pour eux, un acte qui relève du viol de la terre. Les deux agronomes commentent, devant la caméra de Coline Serreau, la qualité d'un sol où la culture du blé est pratiquée de manière intensive. Le tracteur et sa charrue à cinq socs passe sur une terre où le blé vient d'être récolté. Lydia remarque que les sols ont besoin d'un temps de dégradation pour que les micro-organismes se développent et structurent la terre en une matière grumeleuse et aérée. Cependant, le tracteur de labour passe immédiatement après la récolte et creuse de profonds sillons qui font remonter à la surface une terre bordeaux, si lissée qu'elle luit au soleil. Les strates qui apparaissent ainsi à la surface ont donné l'expression « faire saigner la terre ». La profondeur d'où cette terre est issue, et le lissage de sa texture par les socs de la charrue mécanique, la rendent imperméable à la pluie, et impénétrable et invivable pour les micro-organismes. Après plusieurs années, les sols cultivés de cette façon sont dévitalisés, et ne peuvent plus produire les nutriments nécessaires à la culture des plantes. Des intrants de synthèse seront alors indispensables, ainsi que des semences hybrides, adaptées à la déplétion et à l'artificialité synthétique des sols. 
Lorsque Claude et Lydia Bourguignon témoignent du travail qu'ils font auprès d'agriculteurs pour renverser la logique d'agriculture intensive qui appauvrit les sols et favorise l'érosion et les inondations, ils se heurtent à ce qu'ils qualifient de " mythe du labour ». Là où ils préconisent une réhabilitation du principe de jachère et de rotation des cultures, l'utilisation d'engrais et de semences organiques (par opposition aux intrants de synthèse) et la pratique du semis direct (ne nécessitant pas de labour), ils constatent que "psychologiquement, c'est très dur pour les agriculteurs d'arrêter le labour et puis d'accepter de mettre des graines dans un sol où il y a déjà une végétation, c'est un choc psychologique - comme de faire l'amour à une femme qui ne serait pas vierge, à une certaine époque - c'est moi seulement et personne d'autre qui défonce ma terre et si on laisse le semis direct il va se passer des choses qui vont m'échapper, la terre va devenir sauvage, il y a des choses que je ne vais plus contrôler ${ }^{31}$ ». Claude Bourguignon utilise ainsi la métaphore du viol de la terre pour illustrer à quel point le labour reflète une attitude viriliste et dominatrice vis-à-vis de la terre, en expliquant qu'inciter les agriculteurs à cesser de labourer revient à leur demander « d'arrêter de violer la terre, par où leur virilité s'exprime ${ }^{32}$ ».

Nous voulions dans cet article énoncer en quoi le concept métaphorique du viol de la terre pourrait donner lieu à des analyses écoféministes appliquant la notion de consentement aux formes de vie non-humaines. Johnson et Lakoff, qui encouragent la formulation de nouvelles métaphores, soutiennent que celles-ci « peuvent être appropriées parce qu'elles autorisent des actions, justifient des inférences, et nous aident à fixer des objectifs ${ }^{33}$ ». En l'occurrence, les inférences de ce concept métaphorique portent sur une forme de surdité développée par les sociétés occidentales vis-à-vis de ce que Roszak nomme la voix de la terre - The Voice of the Earth - titre d'un de ses ouvrages. La métaphore du viol de la terre indique également qu'une coopération entre sciences humaines et sciences naturelles est indispensable, en particulier pour comprendre ce qui lie les humains à leur habitat, et ce qui influence leur comportement.

La métaphore du viol de la terre vise à appuyer des réflexions et des actions anti-spécistes qui dénoncent l'absence de consentement des formes de vie non-humaines et défendent leur intégrité. La culture du viol a récemment été identifiée comme un phénomène sociologique doté d'une théorisation approfondie et abondamment (bien qu'insuffisamment) relayée, au sujet de la nécessité d'une éducation à la prise en compte du consentement de l'autre

31 Claude et Lydia Bourguignon, propos recueillis par Colline Serreau dans Solutions Locales pour Désordre Global, France, Mémento Films, 2010.

32 Id.

33 Mark Johnson et George Lakoff, Les métaphores dans la vie quotidienne, op. cit., p. 151. 
dans tous les aspects des relations sociales. Par extension, il s'agit de soutenir la proposition formulée par le concept d'écopsychologie développé par Roszak, selon lequel l'équilibre psychique humain dépend d'une relation saine avec l'environnement planétaire au sens large.

Enfin, un des objectifs immédiats que cette métaphore peut permettre d'atteindre est de ne laisser aucun doute sur la corrélation entre la misogynie affichée de Donald Trump et son choix d'une agence fédérale pour la protection de l'environnement - Environmental Protection Agency - qui nie l'origine humaine des dérèglements climatiques actuels, affirmant ainsi sa conviction de la suprématie du patriarcat blanc sur toutes autres formes de vie.

\section{Noémie Moutel}

ERIBIA

Université de Caen Normandie noemie.moutel@unicaen.fr

\section{Résumé}

Le concept métaphorique que cet article propose d'étudier est celui du viol de la terre. C'est à la lumière des constats écoféministes contemporains, qui soulignent la consubstantialité de l'exploitation des femmes et des ressources naturelles, qu'il nous semble pertinent d'observer la récurrence de cette métaphore dans les représentations culturelles occidentales. Il s'agit ainsi de rendre saillant un mode de pensée et d'action caractéristique du néo-libéralisme blanc et patriarcal.

\section{Mots-clés}

Écoféminisme, métaphore, terre-comme-femme, Theodore Roszak, viol.

\section{Abstract}

The metaphorical concept that this article tackles is that of the rape of the earth. In the continuity of contemporary ecofeminist assessments, that underline the consubstantiality of the exploitation of women and natural resources, it seems relevant to observe the recurrence of this metaphor in western cultural representations. The objective is thus to shed light on a prominent mode of thinking and acting which is characteristic of white patriarcal neo-liberalism.

\section{Keywords}

Ecofeminism, Metaphor, Land-as-woman, Theodore Roszak, Rape. 